\title{
Baby de Sitter black holes and $\mathrm{dS}_{3} / \mathrm{CFT}_{2}$
}

\author{
Sophie de Buyl, ${ }^{a}$ Stéphane Detournay, ${ }^{a}$ Gaston Giribet ${ }^{b, c}$ and Gim Seng $\mathbf{N g}^{a}$ \\ ${ }^{a}$ Center for the Fundamental Laws of Nature, Harvard University, \\ Cambridge, MA 02138, U.S.A. \\ ${ }^{b}$ IFIBA-CONICET and Physics Department, University of Buenos Aires, \\ Buenos Aires, 1428 Argentina \\ ${ }^{c}$ Instituto de Física, Pontificia Universidad Católica de Valparaíso, \\ Casilla 4950, Valparaiso, Chile \\ E-mail: sdebuyl@physics.harvard.edu, sdetourn@physics.harvard.edu, \\ gaston@df.uba.ar, gng@fas.harvard.edu
}

ABSTRACT: Unlike three-dimensional Einstein gravity, three-dimensional massive gravity admits asymptotically de Sitter space (dS) black hole solutions. These black holes present interesting features and provide us with toy models to study the dS/CFT correspondence. A remarkable property of these black holes is that they are always in thermal equilibrium with the cosmological horizon of the space that hosts them. This invites us to study the thermodynamics of these solutions within the context of dS/CFT. We study the asymptotic symmetry group of the theory and find that it indeed coincides with the local twodimensional conformal algebra. The charge algebra associated to the asymptotic Killing vectors consists of two copies of the Virasoro algebra with non-vanishing central extension. We compute the mass and angular momentum of the dS black holes and verify that a naive application of Cardy's formula exactly reproduces the entropy of both the black hole and the cosmological horizon. By adapting the holographic renormalization techniques to the case of dS space, we define the boundary stress tensor of the dual Euclidean conformal field theory.

KEYwORDS: dS vacua in string theory, AdS-CFT Correspondence

ARXIV EPRINT: 1308.5569 


\section{Contents}

1 Introduction 1

2 Massive gravity in de Sitter space $\quad 4$

2.1 Three-dimensional massive gravity 4

$\begin{array}{ll}2.2 & \text { Phase space, symmetries and charges } \\ \end{array}$

3 Black holes in de Sitter space $\quad 8$

3.1 Static black holes 8

3.2 Rotating black holes in dS 9

$\begin{array}{lll}3.3 & \text { Boundary stress-tensor } & 11\end{array}$

4 Thermodynamics $\quad 13$

$\begin{array}{lll}4.1 & \text { The } \mathrm{dS}_{3} \text { black hole thermodynamics } & 13\end{array}$

$\begin{array}{lll}4.2 & \text { Extremal limit } & 15\end{array}$

$\begin{array}{lll}4.3 & \text { Local thermodynamic stability } & 15\end{array}$

$\begin{array}{lll}5 & \text { Discussion } & 17\end{array}$

\section{Introduction}

Three-dimensional gravity has shown to be a fruitful playground to study the AdS/CFT holographic correspondence and its ramifications. In particular, its features point at the universality of statistical black hole entropy derivations when an $\mathrm{AdS}_{3}$ factor is involved [1]. It also appears as a promising path to explore non-AdS holography, such as anisotropic scale-invariant condensed-matter models [2, 3], Flat Space holography [4-7] and Warped $\mathrm{AdS}_{3}$ spaces $[8,9]$. Three-dimensional gravity has also played a special role in relation to the dS/CFT correspondence [10-16]. It is the aim of this paper to further explore this direction.

The dS/CFT correspondence [10-16] was proposed with the aim of extending the idea of holography to asymptotically de Sitter space. This is certainly a problem of principal importance since we might actually live in such a universe! The main idea is that quantum gravity on $d$-dimensional $\mathrm{dS}$ space could be in correspondence with a $(d-1)$-dimensional Euclidean conformal field theory. More precisely, mimicking what happens in AdS/CFT, a minimal version of the dS/CFT conjecture states that perturbative correlation functions in $\mathrm{dS}_{d}$ with appropriate future boundary conditions $[17,18]$ on future null infinity $\mathcal{I}^{+}$are given by correlation functions of a $(d-1)$-dimensional Euclidean conformal field theory on $\mathcal{I}^{+}$.

In general, extracting precise information from holography is a non-trivial task. This is even more so in $\mathrm{dS} / \mathrm{CFT}$ partly due to the fact that one still does not have a good 
definition of relevant observables in dS space. ${ }^{1}$ There are, however, some examples in which the holographic dictionary can be established in a more precise way: for instance, a microscopic realization of $\mathrm{dS}_{4} / \mathrm{CFT}_{3}$ was proposed [22] in the context of higher-spin gravity on $\mathrm{dS}_{4}$, relating Vasiliev's theory in $\mathrm{dS}_{4}$ to the dual $\mathrm{Sp}(\mathrm{N}) \mathrm{CFT}_{3}$. A long-anticipated feature (rather than a bug) of dS/CFT - non-unitarity - indeed shows up in this example as the $\mathrm{Sp}(\mathrm{N})$ theory is non-unitary. Even so, the $\mathrm{Sp}(\mathrm{N})$ model retains some rather curiously nice features, one of which is the fact that the appropriate norm (which is conserved by time evolution) in the Hilbert space involves not the standard adjoint but the so-called pseudounitary C-norm [18]. Such implications have been explored in refs. [18, 23] whose work suggest that perhaps one should instead try to understand quantum gravity on de Sitter (which of course includes de Sitter entropy) through appropriate quantization of the quasinormal modes. A surprising and sharp statement that follows is an interesting nonperturbative dS exclusion principle $[18,24]$ which states that one is not allowed to put more than a certain finite quanta of a given quasinormal mode. This exclusion principle may eventually be related to the finiteness of de Sitter entropy. We would also like to highlight that the anti-commuting feature (which makes the $\mathrm{Sp}(\mathrm{N})$ model non-unitary) is very crucial in arguing for the existence of this exclusion principle. In a different direction of understanding the implications of higher-spin $\mathrm{dS}_{4} / \mathrm{CFT}_{3}$, further investigations [25-27] studied the Hartle-Hawking wavefunction of Vasiliev's theory in $\mathrm{dS}_{4}$ and interpreted the results as statements about (in-)stability of $\mathrm{dS}_{4}$ in this theory. Albeit involving a nonunitary $\mathrm{CFT}_{3}$, the higher spin $\mathrm{dS}_{4} / \mathrm{CFT}_{3}$ has (so far) provided a fertile ground in exploring sharp statements about quantum gravity on de Sitter space.

For general $\mathrm{dS}_{d} / \mathrm{CFT}_{d-1}$, on the other hand, one still hopes that symmetry arguments would already provide some interesting physical information. This is particularly realized in the $d=3$ case, ${ }^{2}$ where the conformal group is infinite-dimensional. A particular interesting application of $\mathrm{dS}_{3} / \mathrm{CFT}_{2}$ yields a highly-suggestive derivation of the Kerr- $\mathrm{dS}_{3}$ cosmological horizon entropy in terms of the Cardy formula for the dual $\mathrm{CFT}_{2}[29,30]$.

Besides pure Einstein gravity (GR), other gravitational theories in $2+1$ dimensions (often involving higher-derivative terms) have been explored and have attracted a lot of attention in recent years. An interesting class of such examples includes models of massive gravity. These theories generally contain local propagating degrees of freedom, but this has a price: higher-curvature models usually lead to inconsistencies already at the perturbative level due to the presence of negative-norm (or energy) gravitons. To fix this, one needs to decouple such states coming from extra degrees of freedom due to the higher derivatives. A particularly nice class of theories where such decoupling can be accomplished is the Bergshoeff-Hohm-Townsend (BHT) model [31, 32] (also known as New Massive Gravity or NMG), which amounts to supplementing the Einstein-Hilbert action with special $R^{2}$ terms. Linearized gravitational perturbations in BHT model around flat, $\mathrm{AdS}_{3}$ or $\mathrm{dS}_{3}$ space have been shown to be free of ghost/negative norm states [32] at some special values of the couplings.

\footnotetext{
${ }^{1}$ Nevertheless, see ref. [19-21] for some recent discussions on this issue.

${ }^{2}$ Generalization of the higher-spin $\mathrm{dS} / \mathrm{CFT}$ to $d=3$ case is much less understood, although it has recently been investigated [28].
} 
The general motivation to study the BHT theory of massive gravity is that it provides a toy-model for four-dimensional Einstein gravity. In fact, BHT exhibits several properties that are reminiscent of four-dimensional GR: for instance, in both of these theories there exist two well-behaved local propagating degrees of freedom. Furthermore, in BHT theory there exist interesting non-trivial black holes both in $\mathrm{AdS}_{3}$ and in $\mathrm{dS}_{3}$ spaces [33-35]. These black holes generally have finite mass and angular momentum, together with an extra hair parameter. The causal structure of the $(\mathrm{A}) \mathrm{dS}_{3}$ black holes of BHT theory resembles that of the $(\mathrm{A}) \mathrm{dS}_{4}$ black holes of GR: the Penrose diagrams are similar, and in these geometries there exists a curvature singularity at the origin, shielded by the horizon. Therefore, it provides an interesting playground to apply holographic techniques and try to understand how to interpret hairy black holes with curvature singularities in the dual theory. Some attempts have been made along these lines by studying the scalar wave equation in these backgrounds [36], which can be written as the Heun differential equation. This type of differential equation also appears in studying perturbations about four-dimensional black holes.

As mentioned, in this paper we aim to extend the analysis of $\mathrm{dS}_{3} / \mathrm{CFT}_{2}$ correspondence to the BHT theory and, at the same time, consider black holes in $\mathrm{dS}_{3}$. This analysis will then resemble what might happen in higher-dimensional $\mathrm{dS}_{d} / \mathrm{CFT}_{d-1}$ where black holes do exist. We will study the asymptotic symmetry group of de Sitter space in this theory and show that, indeed, it is generated by two copies of the Virasoro algebra with nontrivial central extensions. This work differs from the $\mathrm{dS}_{3}$ asymptotic symmetries analysis of $[10,11]$ in that we are no longer working in the context of GR. As a consequence, we are considering a more relaxed set of boundary conditions to include the hairy black hole solutions. This results in a different central charge. Despite the more relaxed asymptotic behavior, we will find that the black hole solutions have finite conserved charges which are consistent with the first law of black hole mechanics. One curious feature of these black holes (which already occurred in the $\mathrm{AdS}_{3}$ case [33, 34]) is that they only possess two horizons and both horizons share exactly the same thermodynamical properties. In dS space this implies that the black holes are always in thermal equilibrium with respect to the cosmological horizon. We will find that a naive application of the Cardy formula exactly reproduces the entropy of both the dS cosmological horizon of the black hole horizon.

The paper is organized as follows: in section 2 we discuss three-dimensional massive gravity in asymptotically $\mathrm{dS}$ spaces. We analyze the asymptotic isometry group and show it is generated by two copies of Witt algebra, extending the $\operatorname{sl}(2, \mathbb{C})$ isometry algebra. We also show that the charges associated to the asymptotic Killing vectors close an algebra that turns out to be two copies of Virasoro algebra with negative central extension. In section 3 we consider asymptotically $\mathrm{dS}_{3}$ black hole solutions of BHT theory. These black holes appear at the point of the parameter space at which the BHT theory becomes partially massless. For these solutions we study the conserved charges using both the canonical approach and the quasilocal stress-tensor defined at the boundary of the spacetime. In section 4 we study the thermodynamics both of the $\mathrm{dS}_{3}$ black holes and of the cosmological horizon. We show that the first law of black hole mechanics holds in BHT theory on $\mathrm{dS}_{3}$. Then, we study the extremal limit of these black holes as well as their local thermodynamic (in)stability. We conclude with some speculations in section 5. We first discuss some issues 
related to quantization of the Poisson bracket for $\mathrm{dS}_{3}$ gravity. We then observe that the $\mathrm{dS}_{3}$ black hole thermodynamics potentially has a dual $\mathrm{CFT}_{2}$ interpretation. More precisely, a direct application of the Cardy formula is shown to exactly match both the black hole and the cosmological horizon entropy formulas. This is remarkable since, in addition to mass and angular momentum, the $\mathrm{dS}_{3}$ black holes have an extra hair parameter which eventually combines with the charges in such a way that matching holds.

\section{Massive gravity in de Sitter space}

\subsection{Three-dimensional massive gravity}

Consider the three-dimensional massive gravity theory defined by the action ${ }^{3}$

$$
\begin{aligned}
I= & \frac{1}{16 \pi G} \int_{\Sigma} d^{3} x \sqrt{-g}(R-2 \Lambda)+ \\
& +\frac{1}{32 \pi G \mu} \int_{\Sigma} d^{3} x \sqrt{-g} \epsilon^{\mu \nu \sigma} \Gamma_{\mu \beta}^{\eta}\left(\partial_{\nu} \Gamma_{\eta \sigma}^{\beta}+\Gamma_{\nu \rho}^{\beta} \Gamma_{\eta \sigma}^{\rho}\right)+ \\
& +\frac{1}{16 \pi G m^{2}} \int_{\Sigma} d^{3} x \sqrt{-g}\left(R_{\mu \nu} R^{\mu \nu}-\frac{3}{8} R^{2}\right) .
\end{aligned}
$$

The first line in (2.1) corresponds to the usual Einstein-Hilbert action, while the second line corresponds to the gravitational Chern-Simons term of Topologically Massive Gravity (TMG) [37]; the third line includes the $R^{2}$-terms of the New Massive Gravity (NMG) proposed in ref. [31], hereafter referred to as BHT theory.

Action (2.1) defines a theory of massive gravity in $d=3$ dimensions, which is (perturbatively) ghost-free about its maximally symmetric vacua for some values of the couplings and with appropriate choice of boundary conditions. The field equations coming from (2.1) read

$$
E_{\mu \nu}:=R_{\mu \nu}-\frac{1}{2} R g_{\mu \nu}+\Lambda g_{\mu \nu}+\frac{1}{\mu} C_{\mu \nu}+\frac{1}{2 m^{2}} K_{\mu \nu}=0,
$$

where $C_{\mu \nu}$ is the Cotton tensor

$$
C_{\mu \nu}=\epsilon_{\mu}^{\alpha \beta} \nabla_{\alpha}\left(R_{\beta \nu}-\frac{1}{4} g_{\beta \nu} R\right),
$$

and where the tensor $K_{\mu \nu}$ is given by

$$
\begin{aligned}
K_{\mu \nu}= & 2 \square R_{\mu \nu}-\frac{1}{2} \nabla_{\mu} \nabla_{\nu} R-\frac{1}{2} g_{\mu \nu} \square R+4 R_{\mu \alpha \nu \beta} R^{\alpha \beta}- \\
& \frac{3}{2} R R_{\mu \nu}-R_{\alpha \beta} R^{\alpha \beta} g_{\mu \nu}+\frac{3}{8} R^{2} g_{\mu \nu} .
\end{aligned}
$$

The property that makes the theory defined by action (2.1) of interest is the absence of the ghostly excitation associated to the mode $\square R$. This follows from the fact that tensors (2.3) and (2.4) satisfy the relations

$$
C_{\mu}^{\mu}=0, \quad K_{\mu}^{\mu}=R_{\mu \nu} R^{\mu \nu}-\frac{3}{8} R^{2},
$$

\footnotetext{
${ }^{3}$ Our convention for the epsilon tensor is $\epsilon_{\phi t r}=\sqrt{-g}$.
} 
which makes the dependence in $\square R$ to disappear from the trace of the equations of motion (2.2).

Here, we restrict ourselves to the parity preserving model, i.e. $1 / \mu=0$. Nevertheless, it is worth mentioning that all the solutions we will discuss here persist when the gravitational Chern-Simons term is included [38].

Equations (2.2) admit asymptotically (anti-) de Sitter solutions with effective (A)dS length $l=l_{ \pm}$where

$$
l_{ \pm}^{2} \equiv \frac{1}{2 \Lambda}\left(1 \pm \sqrt{1+\Lambda m^{-2}}\right)
$$

Here, we will be concerned with the case of positive $l$. In that case, the maximally symmetric solution is given by

$$
d s^{2}=-\left(-\frac{r^{2}}{l^{2}}+1\right) d t^{2}+\left(-\frac{r^{2}}{l^{2}}+1\right)^{-1} d r^{2}+r^{2} d \phi^{2},
$$

which corresponds to $\mathrm{dS}_{3}$ space. The six Killing vectors are

$$
\begin{aligned}
& J_{0}^{ \pm}=-\frac{1}{2}\left(l \partial_{t} \pm i \partial_{\phi}\right), \\
& J_{\sigma}^{ \pm}=\frac{1}{2} e^{\frac{\sigma}{l}(t \mp i l \phi)} \sqrt{r^{2}-l^{2}}\left(\sigma \partial_{r}-\frac{r l}{r^{2}-l^{2}} \partial_{t} \mp i \frac{1}{r} \partial_{\phi}\right)
\end{aligned}
$$

for $\sigma= \pm 1$. These vectors generate the $\operatorname{sl}(2, \mathbb{C})$ algebra

$$
\left[J_{1}^{+}, J_{-1}^{+}\right]=2 J_{0}^{+}, \quad\left[J_{0}^{+}, J_{ \pm 1}^{+}\right]=\mp J_{ \pm 1}^{+},
$$

with $\left(J_{n}^{+}\right)^{*}=J_{n}^{-}$and hence the commutators for $J_{n}^{-}$'s are the same as above but with minus superscripts, while the commutators involving one plus and one minus generator are always zero.

In this paper we will be concerned with the following special point in parameter space:

$$
m^{2} l^{2}=-\frac{1}{2}
$$

at which the theory exhibits special features: at (2.10), which happens when $l_{+}^{2}=l_{-}^{2}$ in (2.5) and $\Lambda=-m^{2}$, the BHT theory on dS space becomes partially massless $[39,40]$. In fact, this corresponds to the so-called Deser-Nepomechie point, where massive spin-2 field theory on dS exhibits a symmetry enhancement and its local degrees of freedom are reduced by one [41, 42]. This symmetry enhancement for BHT theory at (2.10) has been studied in $[32,43]$ and its relation to the existence of $\mathrm{dS}_{3}$ black hole has been investigated in [35]. It might be of interest to explore the implications or interpretations of this point in the context of dS/CFT in the future. Here, we will focus on the black hole solutions which exist at (and only at) the special point (2.10).

Notice that since $\Lambda=1 /\left(2 l^{2}\right)>0$, imposing $\Lambda=-m^{2}$ requires a negative value of the coupling constant $1 / \mathrm{m}^{2}$ of the $R^{2}$-terms in (2.1). To this regard, it is convenient to compare our notation with that of ref. [32] where this special point is also considered. Our effective cosmological constant $l^{-2}$ is the $\Lambda$ used in [32], while our cosmological parameter 
$\Lambda$ in the action coincides with the product of parameters $\lambda m^{2}$ in [32]. In [32] a parameter $\sigma= \pm 1$ is introduced multiplying the scalar curvature invariant in the Einstein-Hilbert piece of the action, while in our case we have $\sigma=+1$; nevertheless, we can effectively change from $\sigma=+1$ to $\sigma=-1$ by changing the sign of the Newton constant $G \rightarrow-G$ and simultaneously changing $m^{2} \rightarrow-m^{2}$. In the notation of [32] the partially massless point corresponds to $\lambda=-1$, while for us this means $m^{2} l^{2}=-1 / 2<0$. Notice that the choice $m^{2}<0$ with $\sigma=+1$ is actually consistent with the dS vacuum, cf. eq. (1.11) of [32]. It would be interesting to perform the linear stability analysis about this background, which is not included in that previous analysis.

\subsection{Phase space, symmetries and charges}

The symmetries of asymptotically $\mathrm{dS}_{3}$ spaces are generated by two copies of Witt algebra $[10,11,44]$, whose generators take the form

$$
\begin{aligned}
\ell_{n}^{ \pm} & =-\frac{1}{2} e^{\frac{n}{l}(t \mp l i \phi)}\left(l \partial_{t}-n r \partial_{r} \pm i \partial_{\phi}\right)+\mathcal{O}\left(r^{-1}\right) \\
& =-i e^{\mp i n x^{ \pm}}\left( \pm \partial_{ \pm}+i n r \partial_{r}\right)+\mathcal{O}\left(r^{-1}\right)
\end{aligned}
$$

where $x^{ \pm} \equiv \phi \pm i t / l$. For $n=0, \pm 1$, these coincide with generators $J_{0}^{+}$and $J_{ \pm 1}^{+}$(and the ones with minus superscript) at large $r$ up to order $\mathcal{O}\left(r^{-1}\right)$. These vectors fields satisfy the conjugate relation $\left(\ell_{n}^{+}\right)^{*}=\ell_{n}^{-}$and the Lie commutator $\left[\ell_{n}^{ \pm}, \ell_{m}^{ \pm}\right]=(n-m) \ell_{n+m}^{ \pm}$.

Diffeomorphisms generated by $\ell_{n}^{ \pm}$preserve a set of boundary conditions specified by their deviation with respect to the $\mathrm{dS}_{3}$ metric (2.6); namely $g_{\mu \nu} \rightarrow g_{\mu \nu}+\Delta g_{\mu \nu}$ with

$$
\begin{aligned}
\Delta g_{r r} & =h_{r r}\left(x^{+}, x^{-}\right) r^{-3}+f_{r r}\left(x^{+}, x^{-}\right) r^{-4}+\cdots \\
\Delta g_{r j} & =h_{r j}\left(x^{+}, x^{-}\right) r^{-2}+f_{r j}\left(x^{+}, x^{-}\right) r^{-3}+\cdots \\
\Delta g_{i j} & =h_{i j}\left(x^{+}, x^{-}\right) r+f_{i j}\left(x^{+}, x^{-}\right)+\cdots
\end{aligned}
$$

where $i, j \in\{+,-\}$.

Boundary conditions (2.13) are the $l \rightarrow i l$ analytic continuation of the relaxed $\mathrm{AdS}_{3}$ boundary conditions proposed in [33], in which the $f_{i j}, f_{i r}$, and $f_{r r}$ components correspond to the standard Brown-Henneaux fall-off conditions [45]. To study the hairy black hole solutions (where $h_{i j}, h_{i r}$, and $h_{r r}$ are non-vanishing), we need to include this relaxed set of boundary conditions. In the coordinates employed to describe the $\mathrm{dS}_{3}$ asymptotic conditions in $[10,11]$, the relaxed boundary conditions we will consider correspond to perturbing the $\mathrm{dS}_{3}$ metric $d s^{2} / l^{2}=e^{2 t} d z d \bar{z}-d t^{2}$ with functions obeying the asymptotic behavior $g_{z \bar{z}}=l^{2} e^{2 t} / 2+\mathcal{O}\left(e^{+t}\right), g_{t t}=-l^{2}+\mathcal{O}\left(e^{-t}\right), g_{z z}=\mathcal{O}\left(e^{t}\right), g_{z t}=\mathcal{O}\left(e^{-2 t}\right)$ when $t \rightarrow+\infty$.

It is often convenient to work in Fefferman-Graham-like gauge, in which an asymptotically $\mathrm{dS}_{3}$ metric takes the form

$$
d s^{2}=-\frac{l^{2} d \rho^{2}}{\rho^{2}}+\sum_{p \in \mathbb{N}} g_{i j}^{(p)} \rho^{2-p} d x^{i} d x^{j}
$$


where $g_{ \pm \pm}^{(0)}=0, g_{+-}^{(0)}=l^{2} / 2$. In pure Einstein gravity, the expansion terminates and the most general such solution is known in closed form (see for instance refs. [46, 47]). In particular, the only non-vanishing metric components are

$$
g_{ \pm \pm}^{(2)}=\frac{l}{2} L^{ \pm}\left(x^{ \pm}\right), \quad g_{ \pm \pm}^{(4)}=\frac{1}{4} L^{+} L^{-} .
$$

Note that because of the reality condition on $x^{ \pm}$, the (complex) functions $L^{+}$and $L^{-}$are related by $\left(L^{+}\right)^{*}=L^{-}$in order for the metric to be real.

In other gravity theories, on the other hand, an expansion in integer powers $p$ is not the most general a priori. For instance, in the case of asymptotically AdS spaces in $\mathrm{BHT}$, it was argued in [48] that this expansion is not appropriate to capture all possible solutions, and one can expect a similar situation in dS space. For the solutions we will be interested in, however, it will be sufficient to consider $p \in \mathbb{N}$. In BHT for generic values of the couplings, the equations of motion impose that the only non-vanishing components in the expansion (2.14) satisfy (2.15). At particular values, however, the situation changes. Expanding the equations of motion as

$$
E_{\mu \nu}=\sum_{p \in \mathbb{N}} E_{\mu \nu}^{(p)} \rho^{2-p}
$$

one finds for instance that

$$
E_{i j}^{(1)} \sim g_{i j}^{(1)}\left(m^{2} l^{2}+\frac{1}{2}\right), E_{\rho \rho}^{(6)} \sim g_{+-}^{(2)}\left(m^{2} l^{2}+\frac{1}{2}\right), \cdots
$$

so that, as a consequence, the components $g_{i j}^{(1)}, g_{i j}^{(3)}$ and $g_{+-}^{(2)}$ are no longer forced to vanish at the point $m^{2} l^{2}+1 / 2=0$. Other special points in the parameter space also appear [48], but here we will not focus on those.

Conserved charges in BHT theory can be obtained in the covariant formalism by integrating the one-form potential

$$
k_{\xi}[\delta g, g] \equiv \epsilon_{\phi \mu \nu} k_{\xi}^{[\mu \nu]}[\delta g, g] d \phi,
$$

which depends on an asymptotic Killing vector $\xi$, the background metric $g$, and the linearized metric perturbation $\delta g$. Its explicit expression, which depends only on the equations of motion, is what is called $Q^{\mu \nu}(\xi)$ in eq. (9) and eq. (29) of ref. [49]. The resulting charge takes the form

$$
Q_{\xi}=\int_{\bar{g}}^{g} d \delta g \oint_{S^{\infty}} k_{\xi}[\delta g, g]
$$

where the inner integral integrates over the constant $t$ and $r$ slice at $r \rightarrow \infty$. This expression computes the infinitesimal charge difference between two nearby solutions, $g$ and $g+\delta g$, while the outer integral is performed in the space of parameters.

For the boundary conditions (2.13), the charges associated to the asymptotic Killing vectors $\ell_{n}^{ \pm}$are

$$
\begin{aligned}
Q_{\ell_{n}^{+}} & =\frac{1}{4 \pi G l} \int d \phi e^{-i n x^{+}}\left(g_{++}^{(2)}-\frac{1}{l^{2}} g_{++}^{(1)} g_{+-}^{(1)}\right) \\
Q_{\ell_{n}^{-}} & =\frac{1}{4 \pi G l} \int d \phi e^{i n x^{-}}\left(g_{--}^{(2)}-\frac{1}{l^{2}} g_{--}^{(1)} g_{+-}^{(1)}\right)
\end{aligned}
$$


The non-linear part coming from the non-vanishing $g_{i j}^{(1)}$ metric components has apparently been overlooked in earlier literature. The charges are manifestly finite and integrable. They are also conserved by virtue of the equations of motion

$$
E_{r \pm}^{(5)} \sim \partial_{\mp}\left(g_{ \pm \pm}^{(2)}-\frac{1}{l^{2}} g_{ \pm \pm}^{(1)} g_{+-}^{(1)}\right) .
$$

The charges (2.20) generate through their Poisson bracket an algebra isomorphic to that of the asymptotic Killing vectors, up to possible central extensions. There have been discussions in earlier literature about the reality of such central extensions in the $\mathrm{dS}_{3}$ case, ${ }^{4}$ which depends on the hermiticity conditions satisfied by the generators in relation to the inner product $[13,30]$.

When realized as asymptotically conserved charges $Q_{\ell_{n}^{ \pm}}$, the algebra they generate is represented by a covariant Poisson bracket [51], which (up to field redefinitions) reads

$$
\left\{Q_{\ell_{n}^{ \pm}}, Q_{\ell_{m}^{ \pm}}\right\} \equiv \delta_{\ell_{m}^{ \pm}} Q_{\ell_{n}^{ \pm}}=Q_{\left[\ell_{n}^{ \pm}, \ell_{m}^{ \pm}\right]}+K_{\ell_{n}^{ \pm}, \ell_{m}^{ \pm}}
$$

where the term $K_{\ell_{n}^{ \pm}, \ell_{m}^{ \pm}}$corresponds to a possible central extension, given by

$$
K_{\ell_{n}^{ \pm}, \ell_{m}^{ \pm}}=\oint_{S^{\infty}} k_{\ell_{n}^{ \pm}}\left[\mathcal{L}_{\ell_{m}^{ \pm}} \bar{g}, \bar{g}\right]
$$

which is given by ${ }^{5}$

$$
K_{\ell_{n}^{ \pm}, \ell_{m}^{ \pm}}=\frac{c^{ \pm}}{12}\left(n^{3}-n\right) \delta_{m+n}
$$

with

$$
c^{ \pm}=-\frac{3 l}{2 G}\left(1-\frac{1}{2 m^{2} l^{2}}\right)
$$

We observe that, as it happens in the $\mathrm{AdS}_{3}$ case, the central charges (2.25) receive a contribution $\sim 1 / m^{2}$ coming from the higher-curvature terms. In the limit $m^{2} \rightarrow \infty$ one recovers the central charge of [50] which differs from [10, 11] by a minus sign.

\section{Black holes in de Sitter space}

\subsection{Static black holes}

At the partially massless point (2.10), the theory (2.1) exhibits special properties as we observed on general grounds in the previous section. In particular, at that point the following metric solves the equations of motion (2.2)

$$
d s^{2}=-\frac{\left(r-r_{+}\right)\left(r_{++}-r\right)}{l^{2}} d t^{2}+\frac{l^{2} d r^{2}}{\left(r-r_{+}\right)\left(r_{++}-r\right)}+r^{2} d \phi^{2},
$$

where $r_{+}$and $r_{++}$are two integration constants.

\footnotetext{
${ }^{4}$ See for instance refs. $[21,29,50]$ and references therein.

${ }^{5}$ The precise relation between $K_{\ell_{n}^{ \pm}, \ell_{m}^{ \pm}}$and the central charge of the dual CFT will be discussed in the conclusions, where we comment of the quantization conditions and speculate on the black hole microstates counting.
} 
The black hole solution (3.1) was first derived in ref. [32, 33], and it can be shown to be the most general spherically symmetric solution to the equations of motion of BHT theory in asymptotically dS space. Provided that both $r_{+}$and $r_{++}$are positive, $r_{+}$represents the location of the black hole event horizon while $r_{++} \geq r_{+}$is the cosmological horizon. It is convenient to define the parameters $b$ and $M$ by $r_{++}+r_{+} \equiv b l^{2}, r_{++} r_{+} \equiv 4 M G l^{2}$. They will be related to the black hole mass (see (3.2) below). Empty $\mathrm{dS}_{3}$ space corresponds to the particular case $r_{++}=-r_{+}= \pm l$, while $r_{++}=-r_{+} \neq \pm l$ correspond to non-rotating Kerr-dS $\mathrm{d}_{3}$ solutions (see refs. $[29,30,50]$ for discussions of the latter solution in the context of dS/CFT). For general $r_{++} \neq-r_{+}$, there is a curvature singularity at $r=0$ as can be seen from the Ricci scalar $R=6 / l^{2}-2 b / r$. In dS space, one would like the observer between $r_{+}$ and $r_{++}$not to be exposed to a naked singularity, ${ }^{6}$ since this observer is the natural static observer (where the Killing vector $\partial_{t}$ is timelike). This means that we should insist that $r_{+}$ is real and non-negative. This gives rise to the inequalities $b^{2} l^{2} \geq 16 G M \geq 0$. The Penrose diagram of the $\mathrm{dS}_{3}$ black hole is identical to that of its higher-dimensional analogues.

Using (2.19) one can compute the zero-mode charges $L_{0}^{+} \equiv Q_{\ell_{0}^{+}}$and $L_{0}^{-} \equiv Q_{\ell_{0}^{-}}$, associated to the Killing vectors $\ell_{0}^{ \pm}=-\frac{1}{2}\left(l \partial_{t} \pm i \partial_{\phi}\right)$. The conserved charge $Q_{\partial_{t}} \equiv \mathcal{M}$ associated to solution (3.1) is ${ }^{7}$

$$
\mathcal{M}=-\frac{1}{16 G l^{2}}\left(r_{++}-r_{+}\right)^{2}=M-\frac{b^{2} l^{2}}{16 G} .
$$

Now, let us consider the $\mathrm{dS}_{3}$ metric, which corresponds to $r_{++}=-r_{+}=l$. In this case we obtain the energy difference between $\mathrm{dS}_{3}$ and the massless $(\mathcal{M}=\mathcal{J}=0)$ solution $^{8}$

$$
\left.\Delta E \equiv Q_{\partial_{t}}\right|_{d S_{3}}=-\frac{1}{4 G}
$$

Notice this is consistent with

$$
\frac{c^{ \pm}}{12}=-\frac{l}{4 G}
$$

according to the $\mathrm{CFT}_{2}$ interpretation, due the identification

$$
L_{0}^{+}+L_{0}^{-}=-l Q_{\partial_{t}}
$$

\subsection{Rotating black holes in dS}

One may also provide the black hole (3.1) with angular momentum. In that case the metric acquires an abstruse form: in a system that asymptotes $\mathrm{dS}_{3}$ in its form (2.6) the rotating black hole solution reads

$$
d s^{2}=-N^{2}(r) F(r) d t^{2}+\frac{d r^{2}}{F(r)}+r^{2}\left(d \phi+N^{\phi}(r) d t\right)^{2}
$$

\footnotetext{
${ }^{6}$ For Kerr-dS 3 in pure gravity, however, a naked conical defect is fine since it corresponds to some point-like or small massive object at the origin.

${ }^{7}$ Note that the black hole mass is given by $-\mathcal{M}=-Q_{\partial_{t}}$, cf. eq. (2.8) of [52].

${ }^{8}$ Notice that there is a one-parameter family of solutions with $\mathcal{M}=\mathcal{J}=0$. This is reminiscent of what happens in TMG at the chiral point, where all BTZ solutions with $M_{B T Z}=J_{B T Z}$ have vanishing charges.
} 
where $N(r), N^{\phi}(r)$, and $F(r)$ are functions of the coordinate $r$, given by

$$
F(r)=\frac{\hat{r}^{2}}{r^{2}}\left[-\frac{\hat{r}^{2}}{l^{2}}+\frac{b}{2}(1+\eta) \hat{r}-\frac{b^{2} l^{2}}{16}(1-\eta)^{2}-4 M G \eta\right]
$$

and

$$
N(r)=1-\frac{b l^{2}}{4 \hat{r}}(1-\eta), \quad N^{\phi}(r)=-\frac{a}{2 r^{2}}(4 G M-b \hat{r}),
$$

where $\hat{r}^{2}=r^{2}+2 M G l^{2}(1-\eta)-\left(b^{2} l^{4} / 16\right)(1-\eta)^{2}$ and where $\eta= \pm \sqrt{1+a^{2} / l^{2}}$ is a rotation parameter. Again, these black holes exist only at the partially massless point $m^{2} l_{-}^{2}=m^{2} l_{+}^{2}=-1 / 2$ of the parameter space.

For convenience, we will work with $\hat{r}$ being the radial coordinate where the metric reads

$$
d s^{2}=-N^{2}(\hat{r}) F(\hat{r}) d t^{2}+\frac{\hat{r}^{2} d \hat{r}^{2}}{[r(\hat{r})]^{2} F(\hat{r})}+[r(\hat{r})]^{2}\left(d \phi+N^{\phi}(\hat{r}) d t\right)^{2} .
$$

We will work with $b \geq 0$, since for the opposite sign we could redefine $\hat{r} \rightarrow-\hat{r}$ to absorb the sign. One can see that there is a curvature singularity at

$$
\hat{r}=\hat{r}_{s} \equiv \frac{b}{4}(1-\eta) l^{2}
$$

as can be seen from the Ricci scalar

$$
R=\frac{6}{l^{2}}-\frac{2 b \eta a}{\hat{r}-\hat{r}_{s}} .
$$

The horizons are now located at

$$
\begin{gathered}
\hat{r}_{++}=\frac{1}{4} b(1+\eta) l^{2}+2 l \sqrt{-G \eta\left(M-\frac{b^{2} l^{2}}{16 G}\right)}, \\
\hat{r}_{+}=\frac{1}{4} b(1+\eta) l^{2}-2 l \sqrt{-G \eta\left(M-\frac{b^{2} l^{2}}{16 G}\right)} .
\end{gathered}
$$

If the two horizons are shielding the singularity, i.e. $\hat{r}_{++} \geq \hat{r}_{+} \geq \hat{r}_{s}$, then we have $\eta \geq$ $1, \hat{r}_{s} \leq 0$ and

$$
0 \leq-\left(M-\frac{b^{2} l^{2}}{16 G}\right) \leq \frac{b^{2} \eta l^{2}}{16 G}
$$

In short, we will focus on the following case:

$$
b \geq 0, \quad \eta \geq 1, \quad 0 \leq-\left(M-\frac{b^{2} l^{2}}{16 G}\right) \leq \frac{b^{2} \eta l^{2}}{16 G} .
$$

Introducing $\hat{r}_{\phi} \equiv 4 G M / b$ and $\hat{r}_{0}^{2} \equiv 2(1-\eta) G l^{2} M-b^{2} l^{4}(\eta-1)^{2} / 16=2(1-\eta) G l^{2} M-\hat{r}_{s}^{2}$, we can rewrite the metric in a nicer form

$$
\begin{aligned}
d s^{2}= & -\frac{\left(\hat{r}-\hat{r}_{s}\right)^{2}\left(\hat{r}_{++}-\hat{r}\right)\left(\hat{r}-\hat{r}_{+}\right)}{l^{2}\left(\hat{r}^{2}-\hat{r}_{0}^{2}\right)} d t^{2}+\frac{l^{2} d \hat{r}^{2}}{\left(\hat{r}_{++}-\hat{r}\right)\left(\hat{r}-\hat{r}_{+}\right)} \\
& +\left(\hat{r}^{2}-\hat{r}_{0}^{2}\right)\left[d \phi+\frac{a b\left(\hat{r}-\hat{r}_{\phi}\right)}{2\left(\hat{r}^{2}-\hat{r}_{0}^{2}\right)} d t\right]^{2} .
\end{aligned}
$$


Note that the function $N(r)$ now becomes

$$
N(\hat{r})=1-\frac{\hat{r}_{s}}{\hat{r}},
$$

and we have $\sqrt{-g}=\left(\hat{r}-\hat{r}_{s}\right)$ while the determinant of the induced $(t, \phi)$-metric is $(\hat{r}-$ $\left.\hat{r}_{++}\right)\left(\hat{r}-r_{+}\right)\left(\hat{r}-\hat{r}_{s}\right)^{2} / l^{2}$.

The charges (2.19) of the rotating solution (3.6)-(3.8) are

$$
\begin{aligned}
\mathcal{M} & \equiv Q_{\partial_{t}}=M-\frac{b^{2} l^{2}}{16 G}=-\frac{1}{16 G l^{2} \eta}\left(\hat{r}_{++}-\hat{r}_{+}\right)^{2}, \\
\mathcal{J} & \equiv Q_{\partial_{\phi}}=J+a \frac{b^{2} l^{2}}{16 G}
\end{aligned}
$$

where $J \equiv-a M$ and $\mathcal{J} \equiv-a \mathcal{M}$.

\subsection{Boundary stress-tensor}

In this section, we would like to show that the results for the conserved charges in previous sections are in agreement with the proposal of ref. $[47,50,53]$ where a prescription to define a stress-tensor associated to the boundary dual $\mathrm{CFT}_{2}$ in the context of dS/CFT was given. The idea in $[47,50,53]$ is adapting the holographic renormalization recipe of AdS/CFT $[47,54,55]$ to the case of asymptotically dS spaces. This consists in defining a regularized version of the Brown-York tensor at the boundary. In the case of asymptotically dS spaces, we will focus on the boundary corresponds to the Euclidean surfaces at future null infinity $\mathcal{I}^{+}$.

As in the case of AdS, to apply the method of $[47,50,53]$ one first needs to supplement the gravity action with adequate boundary terms, which eventually yield the correct boundary stress-tensor. In order to do so we will follow the prescription given in [56], which amounts to first rewriting BHT action in an alternative way. That is, consider the action

$$
S_{\mathrm{BHT}}=\frac{1}{16 \pi G} \int_{\Sigma} d^{3} x \sqrt{-g}\left(f^{\mu \nu}\left(R_{\mu \nu}-\frac{1}{2} R g_{\mu \nu}\right)-\frac{1}{4} m^{2}\left(f_{\mu \nu} f^{\mu \nu}-f^{2}\right)\right),
$$

which, apart from the metric, involves an auxiliary symmetric rank-two field $f_{\mu \nu}$. Being non-dynamical, $f_{\mu \nu}$ field can be integrated and the result plugged back into (3.18). This can be easily shown to reproduce the original BHT action. However, the alternative form for the action (3.18) results convenient to write down the boundary terms.

According to the prescription in [56], boundary terms, $S_{\mathrm{B}}$, are to be introduced in the action for the variational principle to be defined in such a way that both the metric $g_{\mu \nu}$ and the auxiliary field $f_{\mu \nu}$ are fixed on the boundary $\partial \Sigma$. Here, we study asymptotically dS in the future, where the boundary corresponds to future null infinity $\mathcal{I}^{+}$.

With this prescription, the boundary action $S_{\mathrm{B}}$ reads

$$
S_{\mathrm{B}}=-\frac{1}{8 \pi G} \int_{\mathcal{I}^{+}} d^{2} x \sqrt{|\gamma|}\left(K+\frac{1}{2} \hat{f}^{i j}\left(K_{i j}-\gamma_{i j} K\right)\right),
$$

where $\gamma_{i j}$ are the components of the induced metric, $\gamma$ its determinant, and $K_{i j}$ is the extrinsic curvature on $\mathcal{I}^{+}$. We shall define $\hat{f}^{i j}$ below. 
The induced metric $\gamma_{i j}$ of the boundary has components $i, j \in\{t, \phi\}$ and is Euclidean. Note that $\partial_{t}$ is timelike inside the static patch (i.e. inside the cosmological horizon seen by an inertial observer in dS space) while it is spacelike outside the static patch. In particular, it is spacelike at $\mathcal{I}^{+}$which is at large $r \gg l$; see [50]. One considers the following ADM like coordinates

$$
d s^{2}=-N^{2} d r^{2}+\gamma_{i j}\left(d x^{i}+N^{i} d r\right)\left(d x^{j}+N^{j} d r\right),
$$

where $N^{2}$ is the radial lapse function, and $\gamma_{i j}$ is then defined on constant- $r$ surfaces.

In terms of these coordinates, the field $\hat{f}^{i j}$ appearing in (3.19) comes from decomposing the contravariant auxiliary field $f^{\mu \nu}$ as

$$
f^{\mu \nu}=\left(\begin{array}{cc}
f^{i j} & h^{j} \\
h^{i} & s
\end{array}\right)
$$

and then defining

$$
\hat{f}^{i j} \equiv f^{i j}+2 h^{(i} N^{j)}+s N^{i} N^{j} .
$$

Then, the Brown-York stress-tensor is defined by varying ${ }^{9}$ the action $S_{\mathrm{A}}+S_{\mathrm{B}}$ with respect to the metric $\gamma^{i j}$. That is,

$$
T_{i j}=\left.\frac{2}{\sqrt{|\gamma|}} \frac{\delta S}{\delta \gamma^{i j}}\right|_{r=\mathrm{const}} .
$$

This yields $T^{i j}=T_{\mathrm{GR}}^{i j}+T_{\mathrm{BHT}}^{i j}$, which consists of the standard Israel contribution

$$
T_{\mathrm{GR}}^{i j}=\frac{1}{8 \pi G}\left(K^{i j}-K \gamma^{i j}\right),
$$

supplemented by a contribution coming from the higher-curvature terms [56]

$$
\begin{aligned}
T_{\mathrm{BHT}}^{i j}= & -\frac{1}{8 \pi G}\left(\frac{1}{2} \hat{f} K^{i j}+\nabla^{(i} \hat{h}^{j)}-\frac{1}{2} D_{r} \hat{f}^{i j}+K_{k}^{(i} \hat{f}^{j) k}-\right. \\
& \left.\frac{1}{2} \hat{s} K^{i j}-\gamma^{i j}\left(\nabla_{k} \hat{h}^{k}-\frac{1}{2} \hat{s} K+\frac{1}{2} \hat{f} K-\frac{1}{2} D_{r} \hat{f}\right)\right),
\end{aligned}
$$

where $\hat{h^{i}}=N\left(h^{i}+s N^{i} N^{j}\right), \hat{s}=-N^{2} s$, and $\hat{f} \equiv \gamma_{i j} \hat{f}^{i j}$. The covariant $r$-derivative $D_{r}$ is defined as

$$
\begin{aligned}
D_{r} \hat{f}^{i j} & \equiv-\frac{1}{N}\left(\partial_{r} \hat{f}^{i j}-N^{k} \partial_{k} \hat{f}^{i j}+2 \hat{f}^{k(i} \partial_{k} N^{j)}\right) \\
D_{r} \hat{f} & \equiv-\frac{1}{N}\left(\partial_{r} \hat{f}-N^{k} \partial_{k} \hat{f}\right)
\end{aligned}
$$

In order to define the stress-tensor at the boundary of the space it is necessary to regularize the divergence that appears in large $r$ limit. As in the case of asymptotically $\mathrm{AdS}_{3}$

\footnotetext{
${ }^{9}$ In this auxiliary field formalism, the appropriate independent field at the boundary is $f_{i}{ }^{j}$, i.e. the variation of $\gamma^{i j}$ acts trivially on $f_{i}{ }^{j}$. For more details, see [56].
} 
spaces, this is achieved by adding boundary terms constructed out of intrinsic boundary quantities. Such terms have the generic form

$$
S_{\mathrm{C}}=\frac{1}{8 \pi G} \int_{\mathcal{I}^{+}} d^{2} x \sqrt{|\gamma|}\left(\alpha_{0}+\alpha_{1} \hat{f}+\alpha_{2} \hat{f}^{2}+\beta_{2} \hat{f}_{i j} \hat{f}^{i j}+\ldots\right)
$$

where $\alpha_{i}, \beta_{i}$, etc are constant coefficients.

The regularized boundary stress-tensor is then defined by taking the $r \rightarrow \infty$ limit of the improved stress-tensor

$$
T_{i j} \rightarrow T_{i j}^{(\mathrm{reg})}=T_{i j}+\frac{2}{\sqrt{|\gamma|}} \frac{\delta S_{\mathrm{C}}}{\delta \gamma^{i j}}
$$

In the present case of $m^{2} l^{2}=-1 / 2$, where the hairy black holes exists, the choice of counterterms (3.27) that makes the action (and both the mass and angular momentum) finite is $\alpha_{1}=-1 / l$, with all the other coefficients $\alpha_{i \neq 1}, \beta_{i}$, etc set to zero. Since for the case of $\mathrm{AdS}_{3}$ hairy rotating black holes (as discussed in [57]) one prescribes $\alpha_{1}=1 / l_{\text {AdS }}$, the above result for $\mathrm{dS}_{3}$ is consistent with generalizing the prescription discussed in [47] where the counter-terms in dS are the same as that in AdS but with coefficients of opposite signs.

With this regularized stress-tensor defined at the boundary one proposes the following definition of conserved charges

$$
Q_{\xi}=\int d s u^{i} T_{i j}^{(\mathrm{reg})} \xi^{j},
$$

where $d s$ is the line element of the constant- $t$ surfaces at $\partial \Sigma$ (recall $t$ is spacelike at the boundary), $u$ is a unit vector orthogonal to the constant- $t$ surfaces, and $\xi$ is the Killing vector that generates the isometry on $\mathcal{I}^{+}$.

As a result, one finds that the conserved charges of the rotating solution (3.6)-(3.8) are

$$
Q_{\partial_{t}}=M-\frac{b^{2} l^{2}}{16 G}, \quad Q_{\partial_{\phi}}=J+a \frac{b^{2} l^{2}}{16 G}
$$

which exactly match (3.17).

Given the renormalized stress-tensor, one can also follow the holographic renormalization procedure ${ }^{10}$ of $[47,54,55]$ and derive the central charge from the Schwarzian term in the transformation of the (renormalized) stress tensor. For $m^{2} l^{2}=-1 / 2$, this yields $c^{ \pm}=3 l / G$ which, up to a sign, agrees with eq. (2.25). This suggests to define the boundary stress-tensor as $T_{i j} \rightarrow-T_{i j}$ in (3.23), and thus choosing the integration orientation in (3.29) accordingly.

\section{Thermodynamics}

\subsection{The $\mathrm{dS}_{3}$ black hole thermodynamics}

Let us now discuss the black hole thermodynamics in $\mathrm{dS}_{3}$ space, which is actually our principal motivation. Consider first the case of static black holes. The temperatures of

\footnotetext{
${ }^{10}$ For such computations in the context of dS/CFT in pure gravity, see [50, 53].
} 
both the cosmological horizons and of the black hole horizon computed from eq. (3.1) give

$$
T_{+}=T_{++}=\frac{1}{4 \pi l^{2}}\left(r_{++}-r_{+}\right) .
$$

That is, the dS black holes of three-dimensional BHT massive gravity are always in thermal equilibrium with the dS space that hosts them. Moreover, by evaluating the Wald formula one finds that the Bekenstein-Hawking entropy of the black hole $S_{+}$coincides with the Gibbons-Hawking entropy of the cosmological horizon $S_{++}$given by

$$
S_{++}=S_{+}=\frac{\pi}{2 G}\left(r_{++}-r_{+}\right) \text {. }
$$

Now, consider the case of rotating solution (3.6)-(3.8). The temperatures associated to the horizons $\hat{r}_{+}$and $\hat{r}_{++}$read

$$
T_{++}=T_{+}=\frac{\eta}{\pi l} \sqrt{\frac{-2 G \mathcal{M}}{1+\eta}}=\frac{\gamma^{-1}}{4 \pi l^{2}}\left(\hat{r}_{++}-\hat{r}_{+}\right),
$$

where $\gamma \equiv \sqrt{(1+1 / \eta) / 2}$. As in the case of the static black hole (where $\eta=1$ ), the Hawking temperature of the black hole event, $T_{+}$, coincides with the Gibbons-Hawking temperature of the cosmological horizon, $T_{++}$. The entropy associated to the horizon is given by Wald formula which evaluates to be

$$
S_{++}=S_{+}=\pi l \sqrt{-\frac{2 \mathcal{M}}{G}(1+\eta)}=\frac{\pi}{2 G} \gamma\left(\hat{r}_{++}-\hat{r}_{+}\right) .
$$

From the temperature and entropy in eq. (4.3)-(4.4), together with the conserved charges in eq. (3.17), one observes that the first law

$$
-d \mathcal{M}=T_{++} d S_{++}+\Omega_{++} d \mathcal{J},
$$

is satisfied, where

$$
\Omega_{++}=\Omega_{+}=-\frac{1}{l} \sqrt{\frac{\eta-1}{\eta+1}}
$$

The $r_{+}$horizon then satisfies exactly the same relation. Let us define $E_{\mathrm{BH}} \equiv-\mathcal{M}=-Q_{\partial_{t}}$, which is non-negative. Then the black hole horizon satisfies the usual first law

$$
d E_{\mathrm{BH}}=T_{+} d S_{+}+\Omega_{+} d \mathcal{J} .
$$

This definition of the energy of the black hole in dS (in particular the sign) is already used for instance in ref. $[52,58]$ for Kerr- $\mathrm{dS}_{4}$ black holes so that the thermodynamics and charges of the black hole horizon reduces to the standard one in flat space.

Note also that, from [33], we know that there are simple relations between the thermodynamical quantities of the rotating black holes and those of the non-rotating ones. In our convention, we actually have

$$
T_{++}=\gamma^{-1} \sqrt{\eta} \times\left(\left.T_{++}\right|_{\eta=1}\right), \quad S_{++}=\gamma \sqrt{\eta} \times\left(\left.S_{++}\right|_{\eta=1}\right) .
$$

An alternative way of writing the entropy is in terms of thermodynamical quantities:

$$
S_{++}=S_{+}=\frac{2 l^{2} \pi^{2} T_{++}}{G\left(1+l^{2} \Omega_{++}^{2}\right)} .
$$




\subsection{Extremal limit}

Now, let us discuss the extremal limits of the $\mathrm{dS}_{3}$ black hole solutions and their thermodynamics. But let us first consider the rotating $\mathrm{AdS}_{3}$ hairy black holes. They actually have two extremal limits: the extremal rotating limit (like that in Kerr/CFT) and the zero-entropy limit. In fact, in the Kerr/CFT limit, we have self-dual $\mathrm{AdS}_{3}$ while in the zero entropy limit, we get $\mathrm{AdS}_{2} \times S^{1}$. The extremal rotating limit (corresponding to $\eta=0$ ) has no analogue for $\mathrm{dS}$ since $\eta \geq 1$.

Let us focus back on to the dS black holes. The strictly zero entropy limit $\left(r_{+}=r_{++}\right)$ corresponds to $\mathcal{M}=0$ and has a near horizon geometry which is $\mathrm{dS}_{2} \times S^{1}$, already observed in [33] from Euclidean continuation of the instanton $S^{2} \times S^{1}$.

Alternatively, one can consider the Nariai-type limit [52, 58] of the metric in eq. (3.15). First, define the dimensionless near-extremal parameter $\lambda$

$$
\lambda \equiv \frac{\hat{r}_{++}-\hat{r}_{+}}{\hat{r}_{++}}
$$

which is proportional to the temperature of the horizon near extremality

$$
T_{++}=\frac{b(1+\eta)}{16 \pi \gamma} \lambda+\mathcal{O}\left(\lambda^{2}\right)
$$

Now, perform the scaling

$$
\tau=\epsilon k t, \quad x=\frac{\hat{r}_{++}-\hat{r}}{\hat{r}_{++} \epsilon}, \quad \tilde{\phi}=\phi-\Omega_{+} t
$$

taking $\epsilon \rightarrow 0$ with $\lambda / \epsilon$ kept finite, one gets the near-horizon metric

$$
d s^{2}=-\frac{x(\lambda-x)}{l^{2}} d \tau^{2}+\frac{l^{2} d x^{2}}{x(\lambda-x)}+k^{2} d \tilde{\phi}^{2}, \quad k^{2} \equiv \hat{r}_{++}^{2}-\hat{r}_{0}^{2} .
$$

Note that this is just $\mathrm{dS}_{2} \times S^{1}$ where the $\mathrm{dS}_{2}$ temperature is

$$
T_{d S_{2}}=\frac{1}{2 \pi l}
$$

A more familiar coordinate for $\mathrm{dS}_{2}$ can be obtained by $\tau \rightarrow 2 \tau l / \lambda, x \rightarrow \lambda(x / l+1) / 2$ to get

$$
d s^{2}=-\left(-\frac{x^{2}}{l^{2}}+1\right) d \tau^{2}+\left(-\frac{x^{2}}{l^{2}}+1\right)^{-1} d x^{2}+k^{2} d \tilde{\phi}^{2} .
$$

\subsection{Local thermodynamic stability}

Now, let us analyze the local thermodynamical stability of $\mathrm{dS}_{3}$ black holes and show that, as with their other features, it parallels the behavior of higher-dimensional black holes.

Similar to the analysis of $[52,59]$ we can study the stability of the black holes in the canonical ensemble and grand canonical ensemble.

In the canonical ensemble, temperature and angular momentum are fixed while the Helmoltz free energy is given by

$$
F=E-T S,
$$


where $E \equiv E_{\mathrm{BH}}, T \equiv T_{+}$and $S \equiv S_{\mathrm{BH}}$. The heat capacity is

$$
C_{\mathcal{J}}=\left(\frac{\partial E}{\partial T}\right)_{\mathcal{J}}
$$

where we are keeping $\mathcal{J}$ fixed. For the non-rotating black hole, we have that $E(T)=$ $\pi^{2} l^{2} T^{2} / G$ implying that $C_{\mathcal{J}=0}=2 \pi^{2} l^{2} T / G>0$. For the rotating black holes, instead,

$$
E=\frac{\pi^{2} l^{2} T^{2}}{2 G} \times \frac{\eta(E)+1}{\eta(E)^{2}}, \quad \ln (E)=\frac{\sqrt{E^{2} l^{2}+\mathcal{J}^{2}}}{E},
$$

so that

$$
C_{\mathcal{J}}=\left(\frac{2 E \eta^{2}}{T}\right) \times \frac{1}{2-\eta}
$$

which implies that $C_{\mathcal{J}}>0$ whenever $\eta<2$, i.e. $|a|=|\mathcal{J}| / E<\sqrt{3} l$ since the factor in the bracket is non-negative. Note that the heat capacity blows up at $\eta=2$. Thus, we conclude that in the canonical ensemble, the black holes are stable for $|\mathcal{J}|<\sqrt{3} E l$.

On the other hand, in grand canonical ensemble, the system is studied at a fixed temperature and angular velocity, and the thermodynamic potential is given by the Gibbs free energy

$$
G=E-T S-\Omega \mathcal{J}
$$

Local thermodynamic stability is analyzed by studying the Hessian of derivative of the entropy with respect to the charges $H_{i j} \equiv \frac{\partial^{2} S}{\partial y^{i} \partial y^{j}}$ where $y^{i}=(E, \mathcal{J})$. In particular, given the entropy

$$
S(E, \mathcal{J})=\pi \sqrt{\frac{2}{G l}} \sqrt{E l+\sqrt{E^{2} l^{2}+\mathcal{J}^{2}}}
$$

we have a $2 \times 2$ matrix in $(E, \mathcal{J})$

$$
H_{i j}=\left(\begin{array}{ll}
H_{11} & H_{12} \\
H_{12} & H_{22}
\end{array}\right) .
$$

The regions that are locally stable must satisfy

$$
H_{11}<0, \quad H_{22}<0, \quad \operatorname{det} H>0,
$$

i.e. the entropy is at a maximum. In fact,

$$
\operatorname{det} H=-\frac{l \pi^{2}}{4 G\left(E^{2} l^{2}+\mathcal{J}^{2}\right)^{3 / 2}}<0
$$

Thus, similar to the case in Kerr-dS $\mathrm{S}_{4}$ in Einstein gravity analyzed in [52], all black holes are (locally) thermally unstable in the grand canonical ensemble where we allow exchange of angular momentum. 


\section{Discussion}

In this paper, we have studied asymptotically de Sitter black holes in three-dimensional massive gravity. These black holes exhibit several features that are reminiscent of higherdimensional dS black holes in GR. In particular, they possess a curvature singularity at the origin and interesting thermodynamical properties both at the black hole event horizon and at the cosmological horizon. Unlike the GR black holes in $\mathrm{dS}_{d>3}$ space, these $\mathrm{dS}_{3}$ black holes are always in thermal equilibrium with respect with the cosmological horizon of the space that hosts them.

For the massive gravity theory in three-dimensional dS space, we studied the asymptotic isometry group and showed that it is generated by two copies of the local conformal algebra in two-dimensions. The algebra of the charges associated to the asymptotic Killing vectors consists of two copies of Virasoro algebra with negative central charge. We also defined the regularized version of the Brown-York stress-tensor and use it to compute the conserved charges of the black holes.

Now, let us go back to the discussion on the asymptotic symmetry algebra and the algebra of charges associated to it. To understand this, let us first review the situation in $\mathrm{AdS}_{3}$ : in that case, the asymptotic Killing vectors $\tilde{\ell}_{n}$ satisfy

$$
i\left[\tilde{\ell}_{n}^{ \pm}, \tilde{\ell}_{m}^{ \pm}\right]=(n-m) \tilde{\ell}_{n+m}^{ \pm}
$$

and the hermiticity condition

$$
\left(\tilde{\ell}_{n}^{ \pm}\right)^{*}=\tilde{\ell}_{-n}^{ \pm} .
$$

Then, when realized as asymptotically conserved charges $Q_{\tilde{\ell}_{n}^{ \pm}}$, the algebra they generate is represented by a covariant Poisson bracket [51], which (up to field redefinitions) reads

$$
\left\{Q_{\tilde{\ell}_{n}^{ \pm}}, Q_{\tilde{\ell}_{m}^{ \pm}}\right\} \equiv \delta_{\tilde{\ell}_{m}^{ \pm}} Q_{\tilde{\ell}_{n}^{ \pm}}=Q_{\left[\tilde{\ell}_{n}^{ \pm}, \tilde{\ell}_{m}^{ \pm}\right]}+K_{\tilde{\ell}_{n}^{ \pm}, \tilde{\ell}_{m}^{ \pm}},
$$

where the term $K_{\tilde{\ell}_{n}^{ \pm}, \tilde{\ell}_{m}^{ \pm}}$corresponds to a possible central extension, given by (2.23), namely $K_{\tilde{\ell}_{n}^{ \pm}, \tilde{\ell}_{m}^{ \pm}}=\oint_{S^{\infty}} k_{\hat{\ell}_{n}^{ \pm}}\left[\mathcal{L}_{\tilde{\ell}_{m}^{ \pm}} \bar{g}, \bar{g}\right]$. This quantity is imaginary for $\mathrm{AdS}_{3}$. Now, replacing Poisson brackets by commutators and charges by quantum operators, $i\left\{Q_{\tilde{\ell}_{m}^{ \pm}}, Q_{\tilde{\ell}_{n}^{ \pm}}\right\} \rightarrow\left[\tilde{L}_{m}^{ \pm}, \tilde{L}_{n}^{ \pm}\right]$, leads to the expected Virasoro algebra

$$
\left[\tilde{L}_{n}^{ \pm}, \tilde{L}_{m}^{ \pm}\right]=(n-m) \tilde{L}_{n+m}^{ \pm}+\frac{c_{\mathrm{AdS}}}{12}\left(n^{3}-n\right) \delta_{m+n}
$$

with $c_{\text {AdS }}$ being the Brown-Henneaux central charge [45]. The hermiticity condition of the corresponding quantum operators is the standard relation

$$
\left(\tilde{L}_{n}^{ \pm}\right)^{\dagger}=\tilde{L}_{-n}^{ \pm}
$$

which follows from hermiticity of the stress-tensor.

In the $\mathrm{dS}_{3}$ case, in contrast, we have seen that the asymptotic Killing vectors satisfy the Lie commutator

$$
\left[\ell_{n}^{ \pm}, \ell_{m}^{ \pm}\right]=(n-m) \ell_{n+m}^{ \pm}
$$


with the condition

$$
\left(\ell_{n}^{ \pm}\right)^{*}=\ell_{n}^{\mp},
$$

which differs from (5.2). In the context of de Sitter space, on the other hand, the question arises as to what is the natural adjoint relation to impose on the quantum generators. Such questions have been discussed in various works $[13,18,23,30,50]$. In particular, in ref. [30] the different hermiticity conditions are discussed in relation to the different manners of defining an inner product on dS [13]. Here, following [18, 30], we adopt the hermiticity condition (5.5) for $L_{n}^{ \pm}$. Consistency with (5.6)-(5.7) then requires that the quantization rule now becomes ${ }^{11}\left\{Q_{\ell_{m}^{ \pm}}, Q_{\ell_{n}^{ \pm}}\right\} \rightarrow\left[L_{m}^{ \pm}, L_{n}^{ \pm}\right]$.

The unusual quantization rule can be motivated heuristically by thinking in the example of a harmonic oscillator: there, the quantization rule $i\{.,.\} \rightarrow[.,$.$] is equivalent to$ the reality condition on the position (and its conjugate momentum) and the requirement that the corresponding operators to be hermitian. If one formally Euclideanizes time, the corresponding Euclidean momentum $p_{E} \equiv i p$ will satisfy $p_{E}^{*}=-p_{E}$ (complex conjugate acts as Euclidean time reflection), implying that the corresponding quantum operator (for the Euclidean momentum) is naturally anti-hermitian rather than hermitian. This leads to a modified quantization rule of the form $\{.,.\} \rightarrow[.,$.$] . In the present context, we would like$ to suggest that a similar rule is perhaps sensible in de Sitter gravity where the static time outside the horizon is Euclidean. ${ }^{12}$ If one adopts such suggestion, the algebra of quantum operators turns out to be

$$
\left[L_{n}^{ \pm}, L_{m}^{ \pm}\right]=(n-m) L_{n+m}^{ \pm}+K_{\ell_{n}^{ \pm}, \ell_{m}^{ \pm}}
$$

with $K_{\ell_{n}^{ \pm}, \ell_{m}^{ \pm}}$given in (2.23), yielding the central extension (2.25), namely

$$
c^{ \pm}=-\frac{3 l}{2 G}\left(1-\frac{1}{2 m^{2} l^{2}}\right)
$$

which would correspond to the central charge of the dual conformal field theory.

In the case of $m^{2}<0$, which in particular includes the point (2.10) at which the black holes exist, the central charge (2.25) is negative. However, one can always change the sign of $c^{ \pm}$by redefining Virasoro generators as $\ell_{n}^{ \pm} \rightarrow-\ell_{-n}^{ \pm}$. This would map an spectrum for the $\left(L_{0}^{+}+L_{0}^{-}\right)$operator that is bounded from below - see (3.2), (3.5) and (3.14) below into a spectrum that is bounded from above. A convention-independent question is to ask if $c\left(L_{0}^{+}+L_{0}^{-}\right)$is bounded below or above. A usual unitary CFT would have $c\left(L_{0}^{+}+L_{0}^{-}\right)$ bounded below, while in our case this is bounded above.

\footnotetext{
${ }^{11}$ More precisely, consider $\alpha\left\{Q_{\ell_{n}^{ \pm}}, Q_{\ell_{m}^{ \pm}}\right\} \equiv \delta_{\ell_{n}^{ \pm}} Q_{\ell_{m}^{ \pm}}=Q_{\left[\ell_{n}^{ \pm}, \ell_{m}^{ \pm}\right]}+K_{\ell_{n}^{ \pm}, \ell_{m}^{ \pm}}$, and quantization rule $\beta\{Q, Q\} \rightarrow[L, L]$. We have to determine $\alpha$ and $\beta$. Now, taking the complex conjugate of the Poisson bracket $\alpha\left\{Q_{\ell_{n}^{ \pm}}, Q_{\ell_{m}^{ \pm}}\right\}$and using the fact that $Q_{\ell_{n}^{+}}^{*}=Q_{\ell_{n}^{-}}$, we deduce that $\alpha$ is real. On the other hand, taking the dagger of $\alpha\left[L_{n}^{ \pm}, L_{m}^{ \pm}\right]$and using $\left(L_{n}^{ \pm}\right)^{\dagger}=L_{-n}^{ \pm}$we conclude that both $\beta$ and $K_{\ell_{n}^{ \pm}, \ell_{m}^{ \pm}}$are real. This is what led to modifying the quantization rule. We thank G. Compère for helpful discussion on this point.

${ }^{12}$ As expressed in [50], relating a Lorentzian bulk with an Euclidean boundary introduces some extra factors of $i$ and unusual reality conditions which still need to be understood better in order to formulate a correspondence such as dS/CFT.
} 
Notice that a naive Euclidean continuation from $\mathrm{AdS}_{3}$ to $\mathrm{dS}_{3}$ would have led to an imaginary central charge, as pointed out for instance in $[16,29,44,50]$. This differs from the real central charge obtained above as well as from the result from Brown-York stress-tensor. Such difference has to do with the different choice of hermiticity (and hence quantization rule) which is closely related to the convention of stress tensor. One could, alternatively, consider a imaginary central charge $i c^{ \pm}$by appropriately redefining Virasoro generators and try to make sense out of $\mathrm{CFT}_{2}$ computation. Here we will consider a real central charge, which is negative at the special point (2.10). This is what is expected if one closely follows the relationship to $\mathrm{AdS}_{3}$. Indeed, the Virasoro generators in that case read

$$
\tilde{\ell}_{n}^{ \pm}=\frac{1}{2} e^{i n\left(t / l_{\mathrm{AdS}} \pm \phi\right)}\left(l_{\mathrm{AdS}} \partial_{t}-i n r \partial_{r} \pm \partial_{\phi}\right)+\mathcal{O}\left(r^{-1}\right),
$$

where $l_{\mathrm{AdS}}$ is the AdS radius. At the level of Poisson brackets, it is easy to see that under analytic continuation $l_{\mathrm{AdS}} \rightarrow-i l, n \rightarrow-n$, the generators map into $\tilde{\ell}_{n}^{ \pm} \rightarrow i \ell_{n}^{ \pm}$, and hence the Poisson bracket of the $\mathrm{dS}_{3}$ charges satisfy

$$
\left\{Q_{\ell_{n}^{ \pm}}, Q_{\ell_{m}^{ \pm}}\right\}=(n-m) Q_{\ell_{m+n}^{ \pm}}+\frac{c^{ \pm}}{12}\left(n^{3}-n\right) \delta_{n+m},
$$

with $c^{ \pm}$given in (2.25). Hence, the central charge is negative provided one keeps the usual Virasoro structure constants.

It is interesting to observe that the entropy of the black hole solutions (3.1) at the special point $m^{2} l^{2}=-1 / 2$ can be written in the following suggestive form:

$$
S_{++}=\pi \sqrt{-\frac{l}{G}(l \mathcal{M}+i \mathcal{J})}+\pi \sqrt{-\frac{l}{G}(l \mathcal{M}-i \mathcal{J})},
$$

At this point the central charge reads

$$
c^{ \pm}=-\frac{3 l}{G}
$$

and (5.12) can therefore be rewritten as

$$
S_{++}=2 \pi \sqrt{\frac{\left|c^{+}\right| L_{0}^{+}}{6}}+2 \pi \sqrt{\frac{\left|c^{-}\right| L_{0}^{-}}{6}},
$$

with $L_{0}^{ \pm}=-(l \mathcal{M} \mp i \mathcal{J}) / 2$. This manifestly shows that Cardy formula (5.14) exactly reproduces the horizon entropy (4.4). Notice that we implicitly assumed that the effective central charge equals the bare one, by analogy with the $\mathrm{AdS}_{3}$ situation. This is actually a non-trivial assumption which, however, happens to yield the right result.

The absolute value appearing in the Cardy formula is of course non-standard. Let us try to justify it as follows: imagine a $\mathrm{CFT}_{2}$ with real and positive central charge $c^{ \pm}$but with Hamiltonian $\mathcal{M}$ bounded from above instead of from below (this requires sending $\ell_{n} \rightarrow-\ell_{-n}$ with respect to the convention we currently have). Imagine also that for some reason we want to call the state with maximum energy 'the vacuum'. Let us, for simplicity, consider the canonical partition function without angular potential. At finite (positive) 
inverse temperature $\beta$, the usual partition function $\operatorname{Tr} e^{-\beta \mathcal{M}}$ does not exist. However, the quantity $Z=\operatorname{Tr} e^{+\beta \mathcal{M}}$ can be defined, and it allows to compute, for instance, the energy of the system. With $\mathcal{M}_{\mathrm{BH}} \equiv-\mathcal{M}$, the partition function is back at its usual form $Z=\operatorname{Tr} e^{-\beta \mathcal{M}_{\mathrm{BH}}}$, and with $\mathcal{M}_{\mathrm{BH}}=-\partial_{\beta} \log Z, S=\left(1-\beta \partial_{\beta}\right) Z$ we have $d \mathcal{M}_{\mathrm{BH}}=T d S$. In terms of the original Hamiltonian, we thus have $d \mathcal{M}=-T d S$. Of course, this is just saying that when the energy is bounded from above, one can flip the sign of the temperature to make it bounded from below. Furthermore, if $Z$ is the partition function of a $\mathrm{CFT}_{2}$ with positive central charge and $L_{0}=\bar{L}_{0}=l \mathcal{M} / 2$, the usual derivation of the Cardy formula - see e.g. appendix of [9] - will go through if one uses $L_{0}^{\mathrm{BH}}=\bar{L}_{0}^{\mathrm{BH}}=-l \mathcal{M} / 2$, giving a Cardy entropy $S \sim \sqrt{c L_{0}^{\mathrm{BH}}} / 6+\sqrt{c \bar{L}_{0}^{\mathrm{BH}} / 6}=\sqrt{c\left(-L_{0}\right) / 6}+\sqrt{c\left(-\bar{L}_{0}\right) / 6}$. Therefore, in that case, it is natural to have a minus sign in the entropy formula.

Following [30], one can also try to identify the left and right temperatures

$$
T_{L, R} \equiv \frac{T_{++}}{1 \pm i l \Omega_{++}} .
$$

Using eq. (4.9), we have

$$
S_{++}=\frac{\pi^{2} l}{3}\left|c^{ \pm}\right|\left(T_{L}+T_{R}\right)
$$

which is just the canonical ensemble form of Cardy formula. We can see that, in contrast to the $\mathrm{AdS}_{3}$ hairy black hole case, where there exists a extremal rotation limit for which one of these temperatures vanishes [34], here we do not have such situation. It is quite puzzling and perhaps interesting that the $T_{L, R}$ never vanishes except at the special point $\mathcal{M}=0$ where they both vanish (as discussed in section 4.2).

Finally, we showed that a naive application of the Cardy formula in the dual $\mathrm{CFT}_{2}$ theory exactly reproduces the entropy of both the black hole and the cosmological horizon. The fact that Cardy entropy formula matches the entropy of black holes in the bulk of $\mathrm{dS}_{3}$ is quite remarkable. In fact, in addition to mass and angular momentum, these black holes are characterized by an extra hair parameter. Then, the fact that all these parameters conspire in a way the matching holds is quite surprising. Nevertheless, while highly-suggestive, this coincidence is far from being a substantial evidence of dS/CFT. Still, having been able to reproduce the entropy of both the black hole and the cosmological horizon in this setting encourages us to study this type of correspondence further.

\section{Acknowledgments}

The authors thank Dionysios Anninos, Tatsuo Azeyanagi, Alejandra Castro, Geoffrey Compère, Tom Faulkner, Tom Hartman, Diego Hofman, Andrés Goya, Julio Oliva, Soonkeon Nam, Jong-Dae Park, Shahin Sheikh-Jabbari, Andy Strominger, Sang-Heon Yi for useful discussions and comments. S. dB. is funded by the Fonds National de la Recherche Scientifique (Belgium) and also acknowledges Wallonie-Bruxelles International for financial support. S. D. was supported by the Fundamental Laws Initiative of the Center for the Fundamental Laws of Nature, Harvard University. The work of G. G. has been supported by ANPCyT, CONICET, ICTP, NSF, and UBA. G.G. thanks Pontificia Universidad Católica de Valparaíso, where part of this work was done. G. N. was supported in part by DOE grant DE-FG02-91ER40654 and the Fundamental Laws Initiative at Harvard. 
Open Access. This article is distributed under the terms of the Creative Commons Attribution License (CC-BY 4.0), which permits any use, distribution and reproduction in any medium, provided the original author(s) and source are credited.

\section{References}

[1] A. Strominger, Black hole entropy from near horizon microstates, JHEP 02 (1998) 009 [hep-th/9712251] [INSPIRE].

[2] E. Ayón-Beato, G. Giribet and M. Hassaïne, Bending AdS Waves with New Massive Gravity, JHEP 05 (2009) 029 [arXiv:0904.0668] [INSPIRE].

[3] E. Ayón-Beato, A. Garbarz, G. Giribet and M. Hassaïne, Lifshitz Black Hole in Three Dimensions, Phys. Rev. D 80 (2009) 104029 [arXiv:0909.1347] [INSPIRE].

[4] A. Bagchi, S. Detournay and D. Grumiller, Flat-Space Chiral Gravity, Phys. Rev. Lett. 109 (2012) 151301 [arXiv:1208.1658] [INSPIRE].

[5] G. Barnich, A. Gomberoff and H.A. González, The flat limit of three dimensional asymptotically anti-de Sitter spacetimes, Phys. Rev. D 86 (2012) 024020 [arXiv:1204.3288] [INSPIRE].

[6] G. Barnich, Entropy of three-dimensional asymptotically flat cosmological solutions, JHEP 10 (2012) 095 [arXiv:1208.4371] [INSPIRE].

[7] A. Bagchi, S. Detournay, R. Fareghbal and J. Simon, Holography of $3 d$ Flat Cosmological Horizons, Phys. Rev. Lett. 110 (2013) 141302 [arXiv:1208.4372] [INSPIRE].

[8] D. Anninos, W. Li, M. Padi, W. Song and A. Strominger, Warped AdS $S_{3}$ Black Holes, JHEP 03 (2009) 130 [arXiv:0807.3040] [InSPIRE].

[9] S. Detournay, T. Hartman and D.M. Hofman, Warped Conformal Field Theory, Phys. Rev. D 86 (2012) 124018 [arXiv:1210.0539] [InSPIRE].

[10] A. Strominger, The dS/CFT correspondence, JHEP 10 (2001) 034 [hep-th/0106113] [INSPIRE].

[11] A. Strominger, Inflation and the dS/CFT correspondence, JHEP 11 (2001) 049 [hep-th/0110087] [INSPIRE].

[12] C. Hull, Timelike $T$ duality, de Sitter space, large- $N$ gauge theories and topological field theory, JHEP 07 (1998) 021 [hep-th/9806146] [INSPIRE].

[13] E. Witten, Quantum gravity in de Sitter space, hep-th/0106109 [INSPIRE].

[14] D. Klemm, Some aspects of the de Sitter/CFT correspondence, Nucl. Phys. B 625 (2002) 295 [hep-th/0106247] [INSPIRE].

[15] S. Cacciatori and D. Klemm, The Asymptotic dynamics of de Sitter gravity in three-dimensions, Class. Quant. Grav. 19 (2002) 579 [hep-th/0110031] [InSPIRE].

[16] J.M. Maldacena, Non-Gaussian features of primordial fluctuations in single field inflationary models, JHEP 05 (2003) 013 [astro-ph/0210603] [INSPIRE].

[17] D. Anninos, G.S. Ng and A. Strominger, Future Boundary Conditions in de Sitter Space, JHEP 02 (2012) 032 [arXiv:1106.1175] [INSPIRE].

[18] G.S. Ng and A. Strominger, State/Operator Correspondence in Higher-Spin dS/CFT, Class. Quant. Grav. 30 (2013) 104002 [arXiv:1204.1057] [INSPIRE]. 
[19] D. Anninos, S.A. Hartnoll and D.M. Hofman, Static Patch Solipsism: Conformal Symmetry of the de Sitter Worldline, Class. Quant. Grav. 29 (2012) 075002 [arXiv:1109.4942] [INSPIRE].

[20] D. Anninos, de Sitter Musings, Int. J. Mod. Phys. A 27 (2012) 1230013 [arXiv:1205.3855] [INSPIRE].

[21] D. Marolf, I.A. Morrison and M. Srednicki, Perturbative S-matrix for massive scalar fields in global de Sitter space, Class. Quant. Grav. 30, 155023 (2013) [arXiv:1209.6039] [INSPIRE].

$[22]$ D. Anninos, T. Hartman and A. Strominger, Higher Spin Realization of the $d S / C F T$ Correspondence, arXiv:1108.5735 [INSPIRE].

[23] D.L. Jafferis, A. Lupsasca, V. Lysov, G.S. Ng and A. Strominger, Quasinormal Quantization in deSitter Spacetime, arXiv:1305.5523 [INSPIRE].

[24] M. Kleban, G.S. Ng, S. Shenker and A. Strominger, unpublished and in progress.

[25] D. Anninos, F. Denef and D. Harlow, The Wave Function of Vasiliev's Universe - A Few Slices Thereof, Phys. Rev. D 88 (2013) 084049 [arXiv:1207.5517] [InSPIRE].

[26] D. Anninos, F. Denef, G. Konstantinidis and E. Shaghoulian, Higher Spin de Sitter Holography from Functional Determinants, arXiv:1305.6321 [INSPIRE].

[27] S. Banerjee et al., Topology of Future Infinity in dS/CFT, JHEP 11 (2013) 026 [arXiv: 1306.6629] [INSPIRE].

[28] P. Ouyang, Toward Higher Spin dS3/CFT2, arXiv:1111.0276 [INSPIRE].

[29] M.-I. Park, Statistical entropy of three-dimensional Kerr-de Sitter space, Phys. Lett. B 440 (1998) 275 [hep-th/9806119] [INSPIRE].

[30] R. Bousso, A. Maloney and A. Strominger, Conformal vacua and entropy in de Sitter space, Phys. Rev. D 65 (2002) 104039 [hep-th/0112218] [InSPIRE].

[31] E.A. Bergshoeff, O. Hohm and P.K. Townsend, Massive Gravity in Three Dimensions, Phys. Rev. Lett. 102 (2009) 201301 [arXiv:0901.1766] [INSPIRE].

[32] E.A. Bergshoeff, O. Hohm and P.K. Townsend, More on Massive 3D Gravity, Phys. Rev. D 79 (2009) 124042 [arXiv:0905.1259] [INSPIRE].

[33] J. Oliva, D. Tempo and R. Troncoso, Three-dimensional black holes, gravitational solitons, kinks and wormholes for BHT massive gravity, JHEP 07 (2009) 011 [arXiv:0905.1545] [INSPIRE].

[34] G. Giribet, J. Oliva, D. Tempo and R. Troncoso, Microscopic entropy of the three-dimensional rotating black hole of BHT massive gravity,

Phys. Rev. D 80 (2009) 124046 [arXiv: 0909.2564] [INSPIRE].

[35] G. Gabadadze, G. Giribet and A. Iglesias, New Massive Gravity on de Sitter Space and Black Holes at the Special Point, arXiv:1212.6279 [inSPIRE].

[36] Y. Kwon, S. Nam, J.-D. Park and S.-H. Yi, Quasi Normal Modes for New Type Black Holes in New Massive Gravity, Class. Quant. Grav. 28 (2011) 145006 [arXiv:1102.0138] [INSPIRE].

[37] S. Deser, R. Jackiw and S. Templeton, Three-Dimensional Massive Gauge Theories, Phys. Rev. Lett. 48 (1982) 975 [inSPIRE].

[38] J. Oliva, D. Tempo and R. Troncoso, Static spherically symmetric solutions for conformal gravity in three dimensions, Int. J. Mod. Phys. A 24 (2009) 1588 [arXiv:0905.1510] [INSPIRE]. 
[39] S. Deser and R.I. Nepomechie, Gauge Invariance Versus Masslessness in de Sitter Space, Annals Phys. 154 (1984) 396 [INSPIRE].

[40] A. Higuchi, Forbidden Mass Range for Spin-2 Field Theory in de Sitter Space-time, Nucl. Phys. B 282 (1987) 397 [InSPIRE].

[41] S. Deser and A. Waldron, Gauge invariances and phases of massive higher spins in $(A) d S$, Phys. Rev. Lett. 87 (2001) 031601 [hep-th/0102166] [InSPIRE].

[42] S. Deser and A. Waldron, Partial masslessness of higher spins in (A)dS, Nucl. Phys. B 607 (2001) 577 [hep-th/0103198] [INSPIRE].

[43] M. Blagojevic and B. Cvetkovic, Extra gauge symmetries in BHT gravity, JHEP 03 (2011) 139 [arXiv:1103.2388] [InSPIRE].

[44] W.R. Kelly and D. Marolf, Phase Spaces for asymptotically de Sitter Cosmologies, Class. Quant. Grav. 29 (2012) 205013 [arXiv:1202 .5347] [INSPIRE].

[45] J.D. Brown and M. Henneaux, Central Charges in the Canonical Realization of Asymptotic Symmetries: An Example from Three-Dimensional Gravity, Commun. Math. Phys. 104 (1986) 207 [InSPIRE].

[46] C. Krishnan and S. Roy, Higher Spin Resolution of a Toy Big Bang, Phys. Rev. D 88 (2013) 044049 [arXiv: 1305.1277] [INSPIRE].

[47] K. Skenderis, Lecture notes on holographic renormalization, Class. Quant. Grav. 19 (2002) 5849 [hep-th/0209067] [INSPIRE].

[48] C. Cunliff, Non-Fefferman-Graham asymptotics and holographic renormalization in New Massive Gravity, JHEP 04 (2013) 141 [arXiv:1301.1347] [INSPIRE].

[49] S. Nam, J.-D. Park and S.-H. Yi, Mass and Angular momentum of Black Holes in New Massive Gravity, Phys. Rev. D 82 (2010) 124049 [arXiv:1009.1962] [INSPIRE].

[50] V. Balasubramanian, J. de Boer and D. Minic, Mass, entropy and holography in asymptotically de Sitter spaces, Phys. Rev. D 65 (2002) 123508 [hep-th/0110108] [INSPIRE].

[51] G. Barnich and F. Brandt, Covariant theory of asymptotic symmetries, conservation laws and central charges, Nucl. Phys. B 633 (2002) 3 [hep-th/0111246] [INSPIRE].

[52] D. Anninos and T. Anous, A de Sitter Hoedown, JHEP 08 (2010) 131 [arXiv:1002.1717] [INSPIRE].

[53] M. Spradlin, A. Strominger and A. Volovich, Les Houches lectures on de Sitter space, hep-th/0110007 [INSPIRE].

[54] V. Balasubramanian and P. Kraus, A Stress tensor for Anti-de Sitter gravity, Commun. Math. Phys. 208 (1999) 413 [hep-th/9902121] [INSPIRE].

[55] S. de Haro, S.N. Solodukhin and K. Skenderis, Holographic reconstruction of space-time and renormalization in the AdS/CFT correspondence, Commun. Math. Phys. 217 (2001) 595 [hep-th/0002230] [INSPIRE].

[56] O. Hohm and E. Tonni, A boundary stress tensor for higher-derivative gravity in AdS and Lifshitz backgrounds, JHEP 04 (2010) 093 [arXiv: 1001.3598] [INSPIRE].

[57] G. Giribet and M. Leston, Boundary stress tensor and counterterms for weakened $A d S_{3}$ asymptotic in New Massive Gravity, JHEP 09 (2010) 070 [arXiv: 1006.3349] [INSPIRE].

[58] D. Anninos and T. Hartman, Holography at an Extremal de Sitter Horizon, JHEP 03 (2010) 096 [arXiv:0910.4587] [INSPIRE].

[59] D. Birmingham and S. Mokhtari, Thermodynamic Stability of Warped AdS $S_{3}$ Black Holes, Phys. Lett. B 697 (2011) 80 [arXiv:1011.6654] [INSPIRE]. 